\title{
5-ALA-induced fluorescence as a marker for diagnostic tissue in stereotactic biopsies of intracranial lymphomas: experience in 41 patients
}

\author{
Barbara Kiesel, MD, ,2,5 Matthias Millesi, MD, 1,2,5 Adelheid Woehrer, MD, PhD, 2,5 \\ Julia Furtner, MD, PhD, ${ }^{3,5}$ Anahita Bavand, MD, ${ }^{1}$ Thomas Roetzer, MD, ${ }^{2}$ Mario Mischkulnig, MD, ${ }^{1}$ \\ Stefan Wolfsberger, MD, ${ }^{1,5}$ Matthias Preusser, MD, ${ }^{4,5}$ Engelbert Knosp, MD, ${ }^{1,5}$ and \\ Georg Widhalm, MD, PhD',5
}

\begin{abstract}
${ }^{1}$ Department of Neurosurgery, ${ }^{2}$ Institute of Neurology, ${ }^{3}$ Department of Biomedical Imaging and Image-Guided Therapy, ${ }^{4}$ Department of Internal Medicine I, and ${ }^{5}$ Comprehensive Cancer Center-Central Nervous System Tumours Unit, Medical University Vienna, Austria
\end{abstract}

OBJECTIVE Stereotactic needle biopsies are usually performed for histopathological confirmation of intracranial lymphomas to guide adequate treatment. During biopsy, intraoperative histopathology is an effective tool to avoid acquisition of nondiagnostic samples. In the last years, 5-aminolevulinic acid (5-ALA)-induced fluorescence has been increasingly used for visualization of diagnostic brain tumor tissue during stereotactic biopsies. Recently, visible fluorescence was reported in the first cases of intracranial lymphomas as well. The aim of this study is thus to investigate the technical and clinical utility of 5-ALA-induced fluorescence in a large series of stereotactic biopsies for intracranial lymphoma.

METHODS This prospective study recruited adult patients who underwent frameless stereotactic needle biopsy for a radiologically suspected intracranial lymphoma after oral 5-ALA administration. During biopsy, samples from the tumor region were collected for histopathological analysis, and presence of fluorescence (strong, vague, or no fluorescence) was assessed with a modified neurosurgical microscope. In tumors with available biopsy samples from at least 2 different regions the intratumoral fluorescence homogeneity was additionally investigated. Furthermore, the influence of potential preoperative corticosteroid treatment or immunosuppression on fluorescence was analyzed. Histopathological tumor diagnosis was established and all collected biopsy samples were screened for diagnostic lymphoma tissue.

RESULTS The final study cohort included 41 patients with intracranial lymphoma. Stereotactic biopsies with assistance of 5-ALA were technically feasible in all cases. Strong fluorescence was found as maximum level in 30 patients (75\%), vague fluorescence in 2 patients (4\%), and no visible fluorescence in 9 patients (21\%). In 28 cases, samples were obtained from at least 2 different tumor regions; homogenous intratumoral fluorescence was found in 16 of those cases $(57 \%)$ and inhomogeneous intratumoral fluorescence in $12(43 \%)$. According to histopathological analysis, all samples with strong or vague fluorescence contained diagnostic lymphoma tissue, resulting in a positive predictive value of $100 \%$. Analysis showed no influence of preoperative corticosteroids or immunosuppression on fluorescence.

CONCLUSIONS The data obtained in this study demonstrate the technical and clinical utility of 5-ALA-induced fluorescence in stereotactic biopsies of intracranial lymphomas. Thus, 5-ALA can serve as a useful tool to select patients not requiring intraoperative histopathology, and its application should markedly reduce operation time and related costs in the future.

https://thejns.org/doi/abs/10.3171/2018.3.FOCUS1859

KEYWORDS intracranial lymphoma; 5-aminolevulinic acid; stereotactic biopsy; fluorescence; diagnostic tissue

I NTRACRANIAL lymphoma constitutes an important differential diagnosis in the routine neurosurgical practice and accounts for approximately $2 \%-3 \%$ of all brain tumors. ${ }^{14,22,27}$ The majority of intracranial lymphomas occur sporadically as so-called "primary central nervous system lymphoma" (PCNSL), whereas only few arise from systemic disease as secondary intracranial lymphoma.7,11,22 Immunosuppression caused by different factors, such as human immunodeficiency virus (HIV) or organ transplantation regimens, predisposes to intracranial lymphoma

ABBREVIATIONS 5-ALA = 5-aminolevulinic acid; HIV = human immunodeficiency virus; NPV = negative predictive value; PCNSL = primary central nervous system lymphoma; PpIX = protoporphyrin IX; PPV = positive predictive value. SUBMITTED February 1, 2018. ACCEPTED March 22, 2018. INCLUDE WHEN CITING DOI: 10.3171/2018.3.FOCUS1859. 
TABLE 1. Literature overview: 5-ALA-induced fluorescence in intracranial lymphomas

\begin{tabular}{|c|c|c|c|c|c|c|c|c|}
\hline \multirow[b]{2}{*}{ Authors \& Year } & \multirow{2}{*}{$\begin{array}{c}\text { No. of } \\
\text { Pts }\end{array}$} & \multicolumn{4}{|c|}{ Type of Surgery } & \multicolumn{3}{|c|}{ 5-ALA Fluorescence Status } \\
\hline & & Stereotactic Biopsy & Open Biopsy & Endoscopic Biopsy & Resection & Strong (\%) & Vague $(\%)$ & None $(\%)$ \\
\hline Yamaguchi et al., 2007 & 2 & 2 & - & - & - & $2(100)$ & - & - \\
\hline Moriuchi et al., 2011 & 1 & 1 & - & - & - & $1(100)$ & - & - \\
\hline Widhalm et al., 2012 & 7 & 7 & - & - & - & $7(100)$ & - & - \\
\hline von Campe et al., 2012 & 4 & 4 & - & - & - & $4(100)$ & & - \\
\hline Grossman et al., 2014 & 1 & - & - & - & 1 & $1(100)$ & - & - \\
\hline Marbacher et al., 2014 & 8 & 6 & - & - & 2 & $5(62)$ & & $3(38)$ \\
\hline Yamamoto et al., $2015^{29}$ & 1 & - & - & - & 1 & $1(100)$ & - & - \\
\hline Yamamoto et al., $2015^{30}$ & $41(47)^{*}$ & 6 & 27 & 14 & - & $23(56)$ & $11(27)$ & $7(17)$ \\
\hline Evers et al., 2017 & 11 & - & - & - & 11 & $8(73)$ & - & $3(27)$ \\
\hline Present study, 2018 & 41 & 41 & - & - & - & $30(75)$ & $2(4)$ & $9(21)$ \\
\hline
\end{tabular}

Pts = patients.

Values indicate numbers of patients unless otherwise indicated.

* Only 41 patients underwent biopsy using 5-ALA.

development. ${ }^{1,22}$ Generally, the treatment of intracranial lymphomas consists of high-dose methotrexate chemotherapy after histopathological confirmation by neurosurgical biopsy. ${ }^{11}$ Nowadays, navigation-guided frameless stereotactic needle biopsies are frequently performed due to the minimal invasiveness of this procedure. ${ }^{3,7,11}$ Whenever clinically possible, patients with suspected intracranial lymphoma should not receive preoperative corticosteroid treatment to prevent an inaccurate histopathological diagnosis. ${ }^{11,22}$ Although stereotactic lymphoma biopsy constitutes a widely performed standard neurosurgical procedure, it is associated with specific limitations. In this sense, the most important limitation constitutes the acquisition of nondiagnostic biopsy samples, which is reported in up to $29 \%$ of cases. ${ }^{9,31}$ To increase the rate of diagnostic lymphoma biopsies, intraoperative histopathology is frequently performed, but this is time consuming and costly. ${ }^{2,5,10,19}$ Thus, an immediately available marker for diagnostic tumor tissue during stereotactic lymphoma biopsies would be of major importance to overcome these important limitations.

In a prior study comprising 50 patients with different brain tumors, we reported the successful application of 5-aminolevulinic acid (5-ALA)-induced fluorescence in stereotactic needle biopsies for intraoperative confirmation of diagnostic tumor tissue. ${ }^{26}$ In this study, all collected samples showing strong fluorescence contained representative/diagnostic tumor tissue, resulting in a positive predictive value of $100 \% .{ }^{26}$ Interestingly, visible fluorescence was recently observed in the first stereotactic biopsies of intracranial lymphomas as well. ${ }^{15,17,24,26,28,30}$ However, these promising first observations in the literature rely on only a few reports of single cases $^{8,17,28,29}$ or small patient series. ${ }^{15,24,26,30}$ (See Table 1.)

The aim of this study is thus to prospectively investigate the technical and clinical utility of 5-ALA-induced fluorescence in a large patient series of stereotactic needle biopsies for intracranial lymphomas. For this purpose, we intraoperatively assessed the fluorescence status and intratumoral fluorescence homogeneity of the collected biopsy samples. Furthermore, all collected samples were histopathologically screened for diagnostic tumor tissue. Finally, we also analyzed the influence of potential preoperative corticosteroid treatment or immunosuppression on fluorescence in intracranial lymphomas.

\section{Methods}

This prospective study included adult patients $(\geq 18$ years) who underwent stereotactic biopsy using 5-ALA for a radiologically suspected intracranial lymphoma between 2009 and 2017 at the Department of Neurosurgery, Medical University Vienna. The local ethics committee approved this study, and the patients gave informed consent.

\section{Preoperative Imaging and Biopsy Target Definition}

Only patients with radiological suspicion of an intracranial lymphoma according to diagnostic MRI as determined by an experienced neuroradiologist (J.F.) were included. Additionally, contrast-enhanced T1-weighted images were obtained for integration of these data into the navigation system (StealthStation Cranial Treon or S7, Medtronic) as well as for planning and definition of the biopsy target. The contrast-enhancing lesion was defined as biopsy target. In case of multiple lesions, the safest accessible contrast-enhancing lesion was applied as biopsy target. The pattern of contrast enhancement was classified as solid, diffuse, or ring-like. Further, the number of lesions (single or multiple) and biopsy localization were assessed.

\section{Stereotactic Biopsies With Assistance of 5-ALA Fluorescence}

All patients received an oral solution of 5-ALA $(20 \mathrm{mg} /$ $\mathrm{kg}$ body weight) approximately 4 hours before induction of anesthesia. To analyze the intraoperative 5-ALA fluorescence status of the collected biopsy samples, we used a modified neurosurgical microscope (Pentero or NC4, Carl Zeiss Surgical $\mathrm{GmbH}$ ) with violet-blue excitation light in the darkened operating room as described previously. ${ }^{25,26}$ 
In this study, we performed navigation-guided frameless conventional stereotactic biopsies (Vertek Cranial Biopsy set, Medtronic) or robotic stereotactic biopsies (iSYS1 robotic guidance device, iSYS Medizintechnik $\mathrm{GmbH})^{16}$ with assistance of 5-ALA-induced fluorescence as described previously. ${ }^{26}$ In brief, the biopsy needle was inserted into the planned target (contrast-enhancing tumor) and tissue samples were collected; the samples were subsequently checked for visible fluorescence, and the fluorescence status was classified as strong, vague, or no visible fluorescence..$^{20,26}$ Aside from the fluorescence status of each sample, the homogeneity of intratumoral fluorescence (homogenous vs inhomogeneous fluorescence) was additionally assessed in patients with available biopsy samples from at least 2 different regions in the same lymphoma. All fluorescing and nonfluorescing biopsy samples were sent to our neuropathology department for histopathological analysis. Patients were protected from light sources for at least 24 hours after oral administration of 5-ALA in order to prevent potential skin phototoxicity.

\section{Strategy for Intraoperative Histopathology}

In the first period of our study, we routinely performed intraoperative histopathology (frozen section and/or smear preparation) independent of the intraoperative 5-ALA fluorescence status of the collected samples. Due to the promising data of our 5-ALA biopsy study published in $2012,{ }^{26}$ we changed the strategy for intraoperative histopathology at our department and, in general, only demanded intraoperative histopathology in cases of vague or no fluorescence. In contrast, intraoperative histopathology was usually not performed in cases with strong fluorescence.

\section{Histopathological Assessment}

After formalin fixation and paraffin embedding of all biopsy samples, histopathological tumor diagnosis was established in each patient by the local neuropathology team according to the current World Health Organization (WHO) classification on a multi-headed microscope. ${ }^{14,22}$ Furthermore, each collected tissue sample with strong, vague, or no fluorescence was investigated for the presence of tumor tissue diagnostic for lymphoma by an experienced neuropathologist (A.W.). In samples with no visible fluorescence, we also semiquantitatively assessed the tumor cell density (no tumor cells, low, moderate, or high tumor cell density).

\section{Collection of Clinical Data}

To investigate the influence of specific parameters on intraoperative 5-ALA-induced fluorescence, we collected the following clinical data in each patient. 1) Previous history of an extracerebral lymphoma: The presence of a previous lymphoproliferative disease outside the CNS was compiled. 2) Immune status: Immunosuppression due to organ transplantation, HIV infection, or other diseases was investigated to classify each patient as either immunocompetent or immunosuppressed. 3) Intake of corticosteroids: All patients were checked for preoperative intake of corticosteroids at the time of admission to our department (yes or no). In case of preoperative prescription of cor- ticosteroids, we routinely paused the corticosteroid treatment whenever clinically possible for at least 7 days before biopsy. The time period between last corticosteroid dose and biopsy ( $\geq 7$ days or $<7$ days) was documented.

\section{Statistical Analysis}

Statistical analyses were performed using SPSS version 24.0 software (IBM Corp.). Dichotomous variables, such as the immune status, corticosteroid intake, and diagnostic tissue samples, were compared with the intraoperative fluorescence status using chi-square tests. Additionally, sensitivity and specificity, as well as a positive predictive value (PPV) and negative predictive value (NPV) for indication of diagnostic samples depending on the fluorescence status, were calculated. A p value $<0.05$ was considered significant.

\section{Results}

In this prospective study, a total of 44 patients underwent a stereotactic biopsy for a radiologically suspected intracranial lymphoma after 5-ALA administration. In 41 of these 44 patients, the diagnosis of a lymphoma was confirmed by postsurgical histopathological analysis, and thus these patients formed our final study cohort. The remaining 3 patients had to be excluded because they had a histopathological diagnosis other than a lymphoma (2 glioblastomas and 1 leukoencephalitis). None of the included patients experienced any significant side effects attributed to the 5-ALA administration. The stereotactic biopsies with assistance of 5-ALA were technically feasible in all cases. Demographic and clinical characteristics of the final study cohort are presented in Table 2.

\section{5-ALA Fluorescence in Intracranial Lymphomas}

Visible fluorescence was detected in 32 (79\%) of 41 cases during stereotactic biopsy. Strong fluorescence was found as the maximum level in the majority of these cases (30 of 32 cases; 94\%) and vague fluorescence in 2 cases $(6 \%)$. In contrast, no visible fluorescence was noted at all in $9(21 \%)$ of 41 cases. The fluorescence status did not differ significantly between PCNSL (visible fluorescence in $73 \%$ of 30 cases) and secondary cerebral lymphomas (visible fluorescence in $90 \%$ of 11 cases; $p=0.4$ ).

\section{5-ALA Fluorescence Status of Samples and Intratumoral Fluorescence Homogeneity}

In the course of 5-ALA stereotactic biopsies, 104 fluorescing and nonfluorescing tissue samples (median 3, range 1-6) were collected from all 41 patients. In detail, strong fluorescence was found in 52 samples, vague fluorescence in 8 samples, and no visible fluorescence in 44 samples. The homogeneity of intratumoral fluorescence was investigated in 28 patients with available biopsy samples from at least 2 different regions in the same lymphoma. In 16 of these 28 patients (57\%), homogenous intratumoral fluorescence was observed, and in the remaining $12(43 \%)$ intratumoral inhomogeneity of fluorescence was found. In the group of 12 patients with an inhomogeneous intratumoral fluorescence pattern, we found 2 different types of 
TABLE 2. Patient characteristics

\begin{tabular}{|c|c|}
\hline Characteristic & Value \\
\hline No. of pts & $41(100)$ \\
\hline Sex, M:F & $1: 1.2$ \\
\hline \multicolumn{2}{|l|}{ Age in yrs } \\
\hline Median & 66 \\
\hline Range & $34-84$ \\
\hline \multicolumn{2}{|l|}{ Primary CNS lymphoma } \\
\hline Yes & $30(73)$ \\
\hline No & $11(27)$ \\
\hline \multicolumn{2}{|l|}{ Immune status } \\
\hline Immunocompetent & $35(85)$ \\
\hline Immunosuppressed & $6(15)$ \\
\hline PTLD & 3 \\
\hline HIV & 2 \\
\hline Other & 1 \\
\hline \multicolumn{2}{|l|}{ No. of lesions } \\
\hline Multiple lesions & $25(61)$ \\
\hline Single lesion & $16(39)$ \\
\hline \multicolumn{2}{|l|}{ Biopsy localization } \\
\hline Frontal & $10(25)$ \\
\hline Corpus callosum & $7(17)$ \\
\hline Temporal & $6(15)$ \\
\hline Periventricular & $5(13)$ \\
\hline Trigonal & $3(7)$ \\
\hline Central & $3(7)$ \\
\hline Basal ganglia & $3(7)$ \\
\hline Parietal & $2(5)$ \\
\hline Brainstem & $1(2)$ \\
\hline Cerebellar & $1(2)$ \\
\hline \multicolumn{2}{|c|}{ Contrast enhancement on MRI } \\
\hline Solid & $23(56)$ \\
\hline Diffuse & $15(37)$ \\
\hline Ring-like & $3(7)$ \\
\hline \multicolumn{2}{|l|}{ Preop corticosteroids } \\
\hline No & $24(59)$ \\
\hline Yes & $17(41)$ \\
\hline \multicolumn{2}{|l|}{ Lymphoma histology } \\
\hline B-cell lymphoma & $40(98)$ \\
\hline T-cell lymphoma & $1(2)$ \\
\hline
\end{tabular}

PTLD = posttransplant lymphoproliferative disease.

Data are numbers of patients (\%) unless otherwise indicated.

fluorescence within 11 patients (strong/no fluorescence: 6 patients; strong/vague fluorescence: 4 patients; vague/no fluorescence: 1 patient). In the remaining patient, all 3 different fluorescence levels (strong, vague, and no fluorescence) were observed in the same lymphoma.

\section{5-ALA Fluorescence and Diagnostic Tissue Samples}

According to histopathological analysis, all samples with strong or vague fluorescence contained diagnostic tumor tissue. Thus, the PPV of strong or vague fluorescence
TABLE 3. Sensitivity, specificity, positive and negative predictive values

\begin{tabular}{ccc}
\hline & \multicolumn{2}{c}{ Diagnostic Specimens } \\
\cline { 2 - 3 } Factor & $\%$ & $(95 \% \mathrm{Cl})$ \\
\hline Sensitivity & $63 \%$ & $(53-73 \%)$ \\
\hline PPV & $100 \%$ & \\
\hline Specificity & $100 \%$ & $(66-100 \%)$ \\
\hline NPV & $20 \%$ & $(17-25 \%)$ \\
\hline
\end{tabular}

for diagnostic tissue of a lymphoma was $100 \%$. In samples with no visible fluorescence tumor cells were present in 35 cases $(80 \%$; moderate tumor cell density in 8 cases [18\%] and high tumor cell density in 27 cases [62\%]) and absent in 9 cases (20\%). The NPV of 5-ALA-induced fluorescence was $20 \%$, the sensitivity was $63 \%$, and the specificity was $100 \%$. An overview of these data is provided in Table 3, and illustrative cases are shown in Figs. 1 and 2.

In the first period of our study, we routinely performed intraoperative histopathology in each case independent of the 5-ALA fluorescence status of the collected samples; this strategy was applied in a total of 7 cases. All 7 patients showed strong fluorescence as maximum fluorescence level during biopsy, and intraoperative histopathology revealed the presence of diagnostic lymphoma tissue in each case (sensitivity of both methods: $100 \%$ ).

\section{5-ALA Fluorescence and Preoperative Corticosteroids}

At the time of admission to our neurosurgical department, 17 (41\%) of 41 patients had already received treatment with corticosteroids. According to our strategy, treatment with corticosteroids was paused for at least 7 days prior to biopsy in 10 of these 17 cases in which it was considered clinically possible in order to avoid acquisition of nondiagnostic tissue samples. In the remaining 7 patients, clinical condition and urgency of the procedure did not allow termination of corticosteroids (4 of 7 patients) or pause of corticosteroids for at least 7 days ( 3 of 7 patients). To investigate the influence of corticosteroids on fluorescence, we compared the fluorescence status in patients without any corticosteroid treatment or pause for at least 7 days (visible fluorescence in $82 \%$ of 34 patients) to the fluorescence status in patients with ongoing corticosteroid treatment or pause of less than 7 days (visible fluorescence in 57\% of 7 patients). According to these data, we found no influence of preoperative corticosteroid treatment on 5-ALA-induced fluorescence $(\mathrm{p}=0.1)$.

\section{5-ALA Fluorescence and Immunosuppression}

Our cohort of 41 patients included 6 patients $(15 \%)$ with immunosuppression. Of these, 3 patients were immunosuppressed due to prior transplantation (2 cases of kidney transplantation and 1 case of heart transplantation), 2 patients had HIV infection, and 1 patient was receiving long-term corticosteroid treatment for sarcoidosis. During biopsy, strong fluorescence was found in 4 of these patients and no visible fluorescence was detected in the remaining 2 patients. According to our data, we found no influence of immunosuppression on 5-ALA-induced fluorescence $(\mathrm{p}=0.4)$. 

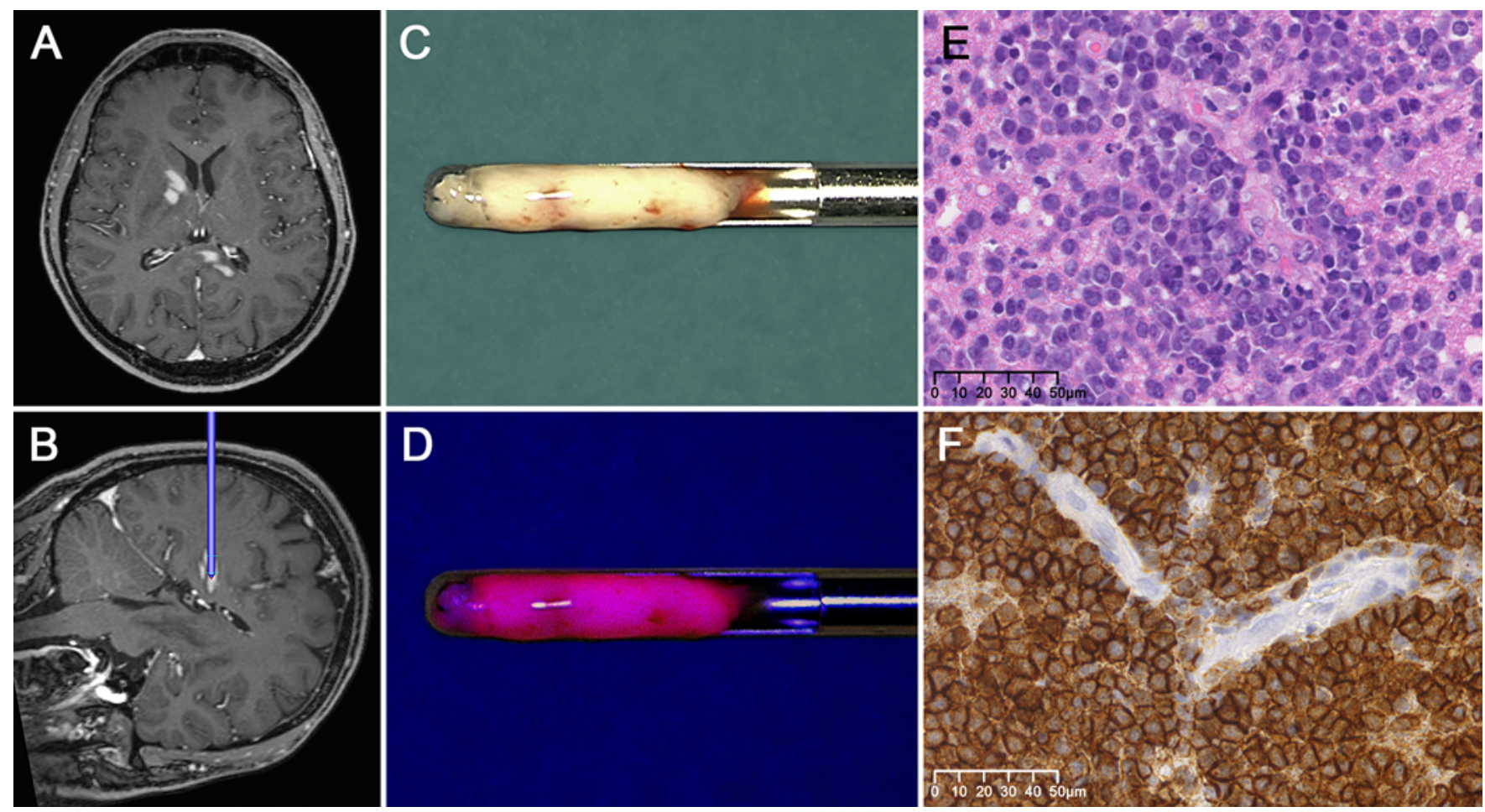

FIG. 1. Stereotactic needle biopsy of an intracranial lymphoma with strong fluorescence. Preoperative MRI demonstrated 2 lesions on $\mathrm{Gd}$-enhanced T1-weighted sequences (A) with diffuse contrast enhancement suspicious for intracranial lymphoma. Since the lesion in the splenium of the corpus callosum was considered to be safer to access the needle was advanced to this enhancing tumor target area during the stereotactic biopsy under navigational guidance (B). The collected tissue sample (C), still inside the biopsy needle, was checked for visible fluorescence with a modified microscope and showed strong fluorescence (D) after the switch to violet-blue excitation light. Therefore, no intraoperative histopathology was requested and the biopsy was terminated. Histopathological analysis of this strongly fluorescing sample revealed characteristics of a diffuse large B-cell lymphoma on $\mathrm{H} \& \mathrm{E}$ staining (E) with CD20-positive cells (F).

\section{Morbidity and Mortality}

During our study period, no surgically related deaths occurred. One patient developed transient hemiparesis after the biopsy as a result of a hemorrhage, which was detected by postoperative CT scan. The hemorrhage did not, however, require surgical removal, and the patient's hemiparesis resolved within 3 months after biopsy. Another patient underwent surgical revision and treatment with antibiotics for a postsurgical wound infection.

\section{Discussion}

In the present study, we prospectively investigated the technical and clinical utility of 5-ALA-induced fluorescence in the largest patient series to date of stereotactic biopsies for intracranial lymphoma. Stereotactic biopsies with assistance of 5-ALA were technically feasible in all of the patients in our cohort, and visible fluorescence was observed in the majority of the lymphomas. Furthermore, all collected samples with visible fluorescence contained diagnostic lymphoma tissue. Therefore, 5-ALA-induced fluorescence is a powerful intraoperative marker for diagnostic tissue in stereotactic biopsies of intracranial lymphomas.

\section{5-ALA Fluorescence in Stereotactic Lymphoma Biopsies}

A previous study of the use of 5-ALA fluorescence in stereotactic biopsies of suspected intracranial lymphomas involved 6 patients, with visible 5-ALA-induced fluorescence in half of the biopsied tumors. ${ }^{15}$ In another study, which mainly focused on open and endoscopic biopsies, but also included some cases of stereotactic biopsies, visible fluorescence was observed in $83 \%$ of intracranial lymphomas. ${ }^{30}$ Additionally, 2 further stereotactic biopsy series, together including a total of 11 patients, found visible fluorescence in all analyzed intracranial lymphomas. ${ }^{25,26}$ In the present stereotactic biopsy series of intracranial lymphomas, the largest such series reported on to date, we detected visible fluorescence in $79 \%$ of 41 patients. This intratumoral fluorescence was homogenous in approximately half of the analyzed lymphomas and inhomogeneous in the remaining half of cases. In the majority of the lymphomas, we observed strong fluorescence (94\%), whereas vague fluorescence was present only in few cases $(6 \%)$. Our data are in accordance with a recent report on open resections demonstrating visible fluorescence in $73 \%$ of 11 lymphoma cases. ${ }^{6}$ Consequently, data from our current study as well as the literature clearly demonstrate that a large number of intracranial lymphomas can be visualized by 5-ALA-induced fluorescence in the course of stereotactic biopsies.

According to our present data, approximately $20 \%$ of intracranial lymphomas lack visible fluorescence during stereotactic biopsies. This observation is in line with other 

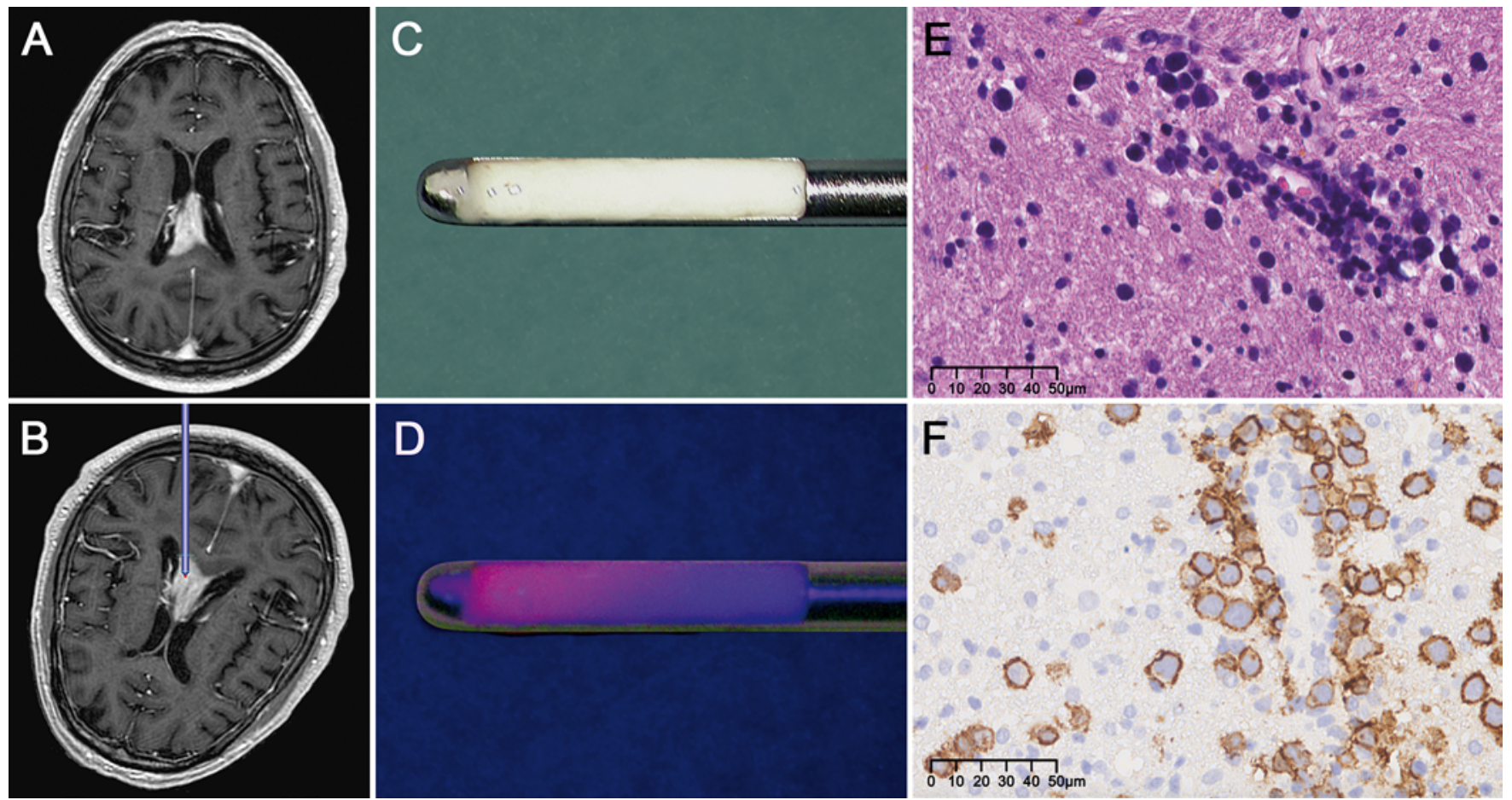

FIG. 2. Stereotactic needle biopsy of an intracranial lymphoma with vague fluorescence. Preoperative MRI (A) demonstrated a single lesion on T1-weighted sequences with solid contrast enhancement suspicious for intracranial lymphoma. The contrastenhancing lesion in the corpus callosum was used as target for stereotactic biopsy under navigational guidance (B). The collected tissue sample still inside the biopsy needle (C) was checked for visible fluorescence and showed vague fluorescence (D). Histopathological analysis of this sample showed a diffuse large B-cell lymphoma on $\mathrm{H} \& \mathrm{E}$ staining $(\mathrm{E})$ with CD20-positive cells $(\mathrm{F})$.

reports in the literature that described intracranial lymphomas with absence of visible fluorescence. ${ }^{6,15,30}$ Since nonfluorescing samples were mainly composed of tumor tissue with moderate and high cell density $(80 \%)$, we believe that the metabolism of 5-ALA is more likely to be responsible for lack of fluorescence than quantitative aspects of lymphoma cell density. The exact mechanism of 5-ALA uptake and its intracellular metabolism to protoporphyrin IX (PpIX), inducing visible fluorescence in intracranial lymphomas, is not fully understood so far. Recently, a proton-coupled folate transporter was described that might play an important role in the metabolism of 5-ALA in lymphomas. ${ }^{4,6,30}$ However, further studies are required in order to clarify the precise mechanism for the presence or absence of 5-ALA-induced fluorescence in intracranial lymphomas.

\section{5-ALA Fluorescence and Diagnostic Lymphoma Tissue}

All biopsy samples in our study with strong or vague fluorescence contained tissue that was histopathologically diagnostic for lymphoma, resulting in a PPV of $100 \%$. Thus, 5-ALA-induced fluorescence is a powerful marker for diagnostic tissue in stereotactic biopsies of intracranial lymphomas that is immediately available and unaffected by brain shift. However, tumor cells were also detected in most samples $(80 \%)$ that lacked visible fluorescence, resulting in a low NPV of $20 \%$. Similarly, low NPVs of 5-ALA-induced fluorescence in the range of $26 \%-51 \%$ were recently described for glioblastomas as well. . $^{12,18,21}$ To overcome this limitation, intraoperative detection of nonfluorescing lymphoma tissue by quantitative analysis of PpIX accumulation with spectroscopic handheld probes might be a promising approach. ${ }^{21,23}$

\section{Influence of Corticosteroids and Immunosuppression on 5-ALA Fluorescence}

A recent in vitro study of glioblastoma cells demonstrated that corticosteroids result in a significantly decreased 5-ALA-induced PpIX production by tumor cells. ${ }^{13}$ Additionally, we hypothesized that immunosuppression might alter the presence of fluorescence in lymphomas. Thus, we also investigated the influence of prior treatment with corticosteroids or immunosuppression on fluorescence. According to our analysis, however, neither corticosteroids nor immunosuppression had a significant influence on fluorescence. To our knowledge, this is the first report that analyzed the influence of corticosteroid treatment and immunosuppression on 5-ALA-induced fluorescence in a large cohort of intracranial lymphomas.

\section{Proposed Strategy for 5-ALA Stereotactic Biopsies of Suspected Lymphoma}

The results of our initial study on 5-ALA stereotactic biopsies in different brain tumors showed that only samples with strong fluorescence always contained representative/diagnostic tumor tissue-not all samples with vague fluorescence. ${ }^{26}$ In lymphomas, in the current study, we found diagnostic tumor tissue also in all samples with vague fluorescence in addition to all strongly fluorescing 
samples. Nevertheless, these data rely only on histopathological analysis of 8 samples with vague fluorescence. In the interest of safety, our proposed strategy is thus to terminate the stereotactic biopsy for a suspected intracranial lymphoma without intraoperative histopathology only in cases of strong tissue fluorescence. This will markedly reduce operation time as well as costs for the operating room and intraoperative histopathology. Moreover, in cases of strong fluorescence, collection of a reduced number of biopsy samples might also result in decreased morbidity/ mortality due to intratumoral hemorrhage. ${ }^{26}$ In all other cases - that is, those with vague or no fluorescence-we still recommend demanding intraoperative histopathology and proceeding according to our previously proposed strategy for 5-ALA stereotactic brain tumor biopsies. ${ }^{26}$

\section{Limitations of This Study and 5-ALA in Lymphoma Biopsies}

1) First of all, the intraoperative semiquantitative assessment of the fluorescence level (strong, vague, or no fluorescence) of each sample by the neurosurgeon is subjective and thus observer dependent. However, this fluorescence classification was only performed by neurosurgeons with large amounts of experience in 5-ALA fluorescence-guided procedures. Furthermore, we recently demonstrated an almost perfect interobserver agreement between several neurosurgeons from our department on the assessment of these fluorescence levels in glioblastomas. ${ }^{12}$ For objective and observer-independent analysis of fluorescence, the quantitative analysis of 5-ALA-induced PpIX accumulation by spectroscopic probes seems to be a promising approach. $^{21,23}$ 2) We did not systematically collect samples from outside the contrast-enhancing lymphoma lesion and are thus not able to make a statement on the presence of potential visible fluorescence also in the peritumoral region. However, in their recent report on 5-ALA fluorescence in PCNSL, Evers et al. found no visible fluorescence in normal brain tissue during lymphoma resection in any of the 8 patients with strongly fluorescing tumors. ${ }^{6} 3$ ) Unfortunately, 5-ALA fluorescence is only able to confirm the presence of diagnostic tumor tissue but cannot be used to make a statement on the specific histological tumor diagnosis. This is a major limitation of 5-ALA in contrast to intraoperative histopathology in stereotactic biopsies. Consequently, the 5-ALA fluorescence method cannot lead to a change of surgical strategy. However, we only rarely change our surgical strategy from stereotactic biopsy to resection based only on findings of intraoperative histology in primarily suspected lymphomas.

\section{Conclusions}

In this largest patient series to date, we found that all 5-ALA stereotactic biopsies were technically feasible and visible fluorescence was present in the majority of intracranial lymphomas. All collected biopsy samples with such visible fluorescence contained tissue diagnostic for lymphoma, resulting in a PPV of $100 \%$. According to the data of this prospective study, 5-ALA-induced fluorescence is thus a powerful intraoperative marker for diagnostic lymphoma tissue in stereotactic biopsies that is directly avail- able in the operation room and unaffected by brain shift. Administration of 5-ALA in cases of suspected lymphoma is thus a useful tool to select patients who do not require intraoperative histopathology. Consequently, the use of 5-ALA should be considered in the future also in stereotactic biopsies for a suspected intracranial lymphoma in order to markedly reduce operation time and related costs.

\section{Acknowledgments}

We thank Brigitte Hammer-Schmiedel for technical assistance, including preparation of tissue specimens, and Ingrid Dobsak for graphical assistance. This study was performed within the $\mathrm{PhD}$ thesis project of Clinical Neuroscience (CLINS) at the Medical University Vienna.

\section{References}

1. Castellano-Sanchez AA, Li S, Qian J, Lagoo A, Weir E, Brat DJ: Primary central nervous system posttransplant lymphoproliferative disorders. Am J Clin Pathol 121:246-253, 2004

2. Dammers R, Schouten JW, Haitsma IK, Vincent AJPE, Kros JM, Dirven CMF: Towards improving the safety and diagnostic yield of stereotactic biopsy in a single centre. Acta Neurochir (Wien) 152:1915-1921, 2010

3. Deckert M, Brunn A, Montesinos-Rongen M, Terreni MR, Ponzoni M: Primary lymphoma of the central nervous system-a diagnostic challenge. Hematol Oncol 32:57-67, 2014

4. Desmoulin SK, Hou Z, Gangjee A, Matherly LH: The human proton-coupled folate transporter: biology and therapeutic applications to cancer. Cancer Biol Ther 13:1355-1373, 2012

5. Dickerman RD, Mittler MA, Morgan JT: Stereotactic brain biopsies and operative complications: technique to further decrease risks. Acta Neurochir (Wien) 147:911-912, 2005

6. Evers G, Kamp M, Warneke N, Berdel W, Sabel M, Stummer $\mathrm{W}$, et al: 5-aminolaevulinic acid-induced fluorescence in primary central nervous system lymphoma. World Neurosurg 98:375-380, 2017

7. Giannini C, Dogan A, Salomão DR: CNS lymphoma: a practical diagnostic approach. J Neuropathol Exp Neurol 73:478-494, 2014

8. Grossman R, Nossek E, Shimony N, Raz M, Ram Z: Intraoperative 5-aminolevulinic acid-induced fluorescence in primary central nervous system lymphoma. J Neurosurg 120:67-69, 2014

9. Haldorsen IS, Espeland A, Larsen JL, Mella O: Diagnostic delay in primary central nervous system lymphoma. Acta Oncol 44:728-734, 2005

10. Hall WA: The safety and efficacy of stereotactic biopsy for intracranial lesions. Cancer 82:1749-1755, 1998

11. Han $\mathrm{CH}$, Batchelor TT: Diagnosis and management of primary central nervous system lymphoma. Cancer 123:43144324, 2017

12. Kiesel B, Mischkulnig M, Woehrer A, Martínez-Moreno M, Millesi M, Mallouhi A, et al: Systematic histopathological analysis of different 5-aminolevulinic acid-induced fluorescence levels in newly diagnosed glioblastomas. J Neurosurg [epub ahead of print October 27, 2017. DOI: 10.3171/2017.4.JNS162991]

13. Lawrence JE, Steele CJ, Rovin RA, Belton RJ Jr, Winn RJ: Dexamethasone alone and in combination with desipramine, phenytoin, valproic acid or levetiracetam interferes with 5-ALA-mediated PpIX production and cellular retention in glioblastoma cells. J Neurooncol 127:15-21, 2016

14. Louis DN, Ohgaki H, Wiestler OD, Cavenee WK (eds): WHO Classification of Tumours of the Central Nervous System, Revised, ed 4. Lyon, France: International Agency for Research on Cancer, 2016 
15. Marbacher S, Klinger E, Schwyzer L, Fischer I, Nevzati E, Diepers M, et al: Use of fluorescence to guide resection or biopsy of primary brain tumors and brain metastases. Neurosurg Focus 36(2):E10, 2014

16. Minchev G, Kronreif G, Martínez-Moreno M, Dorfer C, Micko A, Mert A, et al: A novel miniature robotic guidance device for stereotactic neurosurgical interventions: preliminary experience with the iSYS1 robot. J Neurosurg 126:985-996, 2017

17. Moriuchi S, Yamada K, Dehara M, Teramoto Y, Soda T, Imakita M, et al: Use of 5-aminolevulinic acid for the confirmation of deep-seated brain tumors during stereotactic biopsy. Report of 2 cases. J Neurosurg 115:278-280, 2011

18. Roberts DW, Valdés PA, Harris BT, Fontaine KM, Hartov A, Fan X, et al: Coregistered fluorescence-enhanced tumor resection of malignant glioma: relationships between $\delta$ aminolevulinic acid-induced protoporphyrin IX fluorescence, magnetic resonance imaging enhancement, and neuropathological parameters. Clinical article. J Neurosurg 114:595603, 2011

19. Shooman D, Belli A, Grundy PL: Image-guided frameless stereotactic biopsy without intraoperative neuropathological examination. J Neurosurg 113:170-178, 2010

20. Stummer W, Novotny A, Stepp H, Goetz C, Bise K, Reulen HJ: Fluorescence-guided resection of glioblastoma multiforme by using 5-aminolevulinic acid-induced porphyrins: a prospective study in 52 consecutive patients. J Neurosurg 93:1003-1013, 2000

21. Stummer W, Tonn JC, Goetz C, Ullrich W, Stepp H, Bink A, et al: 5-aminolevulinic acid-derived tumor fluorescence: the diagnostic accuracy of visible fluorescence qualities as corroborated by spectrometry and histology and postoperative imaging. Neurosurgery 74:310-320, 2014

22. Swerdlow SH, Campo E, Harris NL, Jaffe ES, Pileri SA, Thiele J, et al (eds): WHO Classification of Tumours of Haematopoietic and Lymphoid Tissues, ed 4. Lyon, France: International Agency for Research on Cancer, 2008

23. Valdés PA, Leblond F, Kim A, Harris BT, Wilson BC, Fan $\mathrm{X}$, et al: Quantitative fluorescence in intracranial tumor: implications for ALA-induced PpIX as an intraoperative biomarker. J Neurosurg 115:11-17, 2011

24. von Campe G, Moschopulos M, Hefti M: 5-aminolevulinic acid-induced protoporphyrin IX fluorescence as immediate intraoperative indicator to improve the safety of malignant or high-grade brain tumor diagnosis in frameless stereotactic biopsies. Acta Neurochir (Wien) 154:585-588, 2012

25. Widhalm G: Intra-operative visualization of brain tumors with 5-aminolevulinic acid-induced fluorescence. Clin Neuropathol 33:260-278, 2014
26. Widhalm G, Minchev G, Woehrer A, Preusser M, Kiesel B, Furtner J, et al: Strong 5-aminolevulinic acid-induced fluorescence is a novel intraoperative marker for representative tissue samples in stereotactic brain tumor biopsies. Neurosurg Rev 35:381-391, 2012

27. Wöhrer A, Waldhör T, Heinzl H, Hackl M, Feichtinger J, Gruber-Mösenbacher U, et al: The Austrian Brain Tumour Registry: a cooperative way to establish a population-based brain tumour registry. J Neurooncol 95:401-411, 2009

28. Yamaguchi F, Takahashi H, Teramoto A: Photodiagnosis for frameless stereotactic biopsy of brain tumor. Photodiagn Photodyn Ther 4:71-75, 2007

29. Yamamoto J, Kitagawa T, Akiba D, Nishizawa S: 5-aminolevulinic acid-induced fluorescence in cerebellar primary central nervous system lymphoma: a case report and literature review. Turk Neurosurg 25:796-800, 2015

30. Yamamoto T, Ishikawa E, Miki S, Sakamoto N, Zaboronok A, Matsuda M, et al: Photodynamic diagnosis using 5-aminolevulinic acid in 41 biopsies for primary central nervous system lymphoma. Photochem Photobiol 91:1452-1457, 2015

31. Zoeller GK, Benveniste RJ, Landy H, Morcos JJ, Jagid J: Outcomes and management strategies after nondiagnostic stereotactic biopsies of brain lesions. Stereotact Funct Neurosurg 87:174-181, 2009

\section{Disclosures}

Stefan Wolfsberger reports currently serving as an educational consultant and a technological advisory board member for Medtronic.

\section{Author Contributions}

Conception and design: Widhalm, Kiesel, Millesi, Wolfsberger, Preusser, Knosp. Acquisition of data: Kiesel, Woehrer, Furtner, Bavand, Roetzer, Mischkulnig, Preusser. Analysis and interpretation of data: all authors. Drafting the article: Widhalm, Kiesel. Critically revising the article: Widhalm, Kiesel, Millesi, Woehrer, Furtner, Roetzer, Mischkulnig, Wolfsberger, Preusser, Knosp. Reviewed submitted version of manuscript: all authors. Approved the final version of the manuscript on behalf of all authors: Widhalm. Statistical analysis: Widhalm, Kiesel, Millesi, Woehrer, Bavand, Roetzer, Mischkulnig. Administrative/technical/material support: Bavand, Mischkulnig. Study supervision: Widhalm.

\section{Correspondence}

Georg Widhalm: Medical University Vienna, Austria. georg. widhalm@meduniwien.ac.at. 